\title{
Ursolic Acid Promotes Clearance of Trypanosoma cruzi amastigotes in the Host Cell
}

\author{
María Cristina Vanrell ${ }^{1}$, Santiago José Martinez ${ }^{1}$, Lucila Ibel Muñoz ${ }^{2}$, Betiana Nebaí Salassa ${ }^{1}$ \\ and Patricia Silvia Romano*1
}

1 Instituto de Histología y Embriología de Mendoza, Facultad de Ciencias Médicas Universidad Nacional de Cuyo. Facultad de Ciencias Médicas U.N.Cuyo-CONICET, Casilla de correo 56, Mendoza CP 5500, Argentina; vanrellcristina@gmail.com (M.C.V.), santigo.mail@gmail.com (S.J.M.), bnsalassa@gmail.com (B.N.S.), promano@fcm.uncu.edu.ar (P.S.R.)

2 Facultad de Farmacia y Bioquímica, Universidad Juan Agustín Maza. Av. Acceso Este, Lateral Sur 2245, Gllén, Mendoza, Argentina; lucilaimunoz@gmail.com

* Correspondence: promano@fcm.uncu.edu.ar

+ Presented at the 1st International Electronic Conference on Biomolecules: Natural and Bio-Inspired Therapeutics for Human Diseases, 1-13 December 2020; Available online: https://iecbm2020.sciforum.net/.

Received: date; Accepted: date; Published: date

\begin{abstract}
Trypanosoma cruzi is the etiological agent of Chagas disease, which is endemic in Latin America. Ursolic acid (UA) is a natural pentacyclic triterpene which has been shown to reduce the peak of parasitemia in T. cruzi infected mice. Due to UA was described as an inducer of autophagy and having into account that our previous work established the protective role of this process on in vivo infections, we decided to study the possible involvement of UA in the elimination of parasites in macrophages and cardiac cells and its possible mechanism of action.To test this, we infected cells with $T$. cruzi for 24 hours, and then treated the samples with UA $(5-10 \mu \mathrm{M})$ for different times. Our data showed that UA significantly decreased the amount of amastigotes compared to non-treated cells. We also studied the effect of UA on the autophagy response and other possible mechanisms of action we observed that UA induces the autophagy pathway, and that LC3, the marker of autophagy, is recruited around amastigotes, indicating xenophagy of these parasites. A cytotoxic effect was observed on T. cruzi trypomastigotes while epimastigotes displayed more resistance to this drug. Moreover, the production of ROS after 24 hours of treatment is increased on infected cells but, interestingly, UA does not have this effect on non-infected cells. We conclude that this natural compound promotes parasite death through induction of autophagy and other host cell responses.
\end{abstract}

Keywords: Chagas disease; Trypanosoma cruzi; xenophagy; ROS

\section{Introduction}

Chagas disease (CD) or American trypanosomiasis is caused by the protozoan Trypanosoma cruzi (T. cruzi). The World Health Organization (WHO) estimates that 8 to 10 million people are infected worldwide, mostly in Latin America where the disease is endemic. CD is also one of the called neglected tropical diseases, because the low importance that governments and industry gave to these illnesses in the past.

T. cruzi has a biphasic biological cycle that develops in both the insect vector and the mammalian hosts. In the latter, T. cruzi behaves as an obligate intracellular parasite, interacting with vesicular transport pathways that allow it to invade the cell and to establish its replicative niche. (Maria Carmo Pereira Nunes, et al. 2013; Jeannette Guarner, et al. 2019 and João Carlos Pinto Dias, et al. 2017). T. cruzi is capable to infect several classes of host cells. Macrophages are the first line of defense during 
T. cruzi invasion. Macrophages may either suppress T. cruzi replication or afford a favorable environment where it can reproduce and be distributed to other area within the body (Peluffo $G$ et al., 2004; Holzmuller P. et al., 2018). On the other hand, cardiac cells are one of the main target for T. cruzi in which the parasite established its replicative niche to form the amastigote nests that are usually present in the heart of the chronic patients.

Ursolic acid (UA) is a naturally occurring pentacyclic triterpene compound with many biological properties. This compound is widely distributed in nature as in rosemary, basil, medlar, oregano, eucalyptus, and coffee and apple skin. It has been shown that UA displayed anti-inflammatory, antioxidant and anti-cancer activities (Khwaza V et al., 2020 and Chen Z et al., 2020). Moreover, UA exerts potent antiviral (Tohmé MJ et al., 2019) and antiprotozoan action (Bilbao-Ramos P et al., 2020; Son and Lee, 2020). Oral administration of UA reduces the parasitaemia peaks in mice infected with T. cruzi Y strain in an acute model of infection (Daniele da Silva Ferreira, et al. 2013). Interestingly, UA has been described as an autophagy inducer in cancer cells (Lin JH et al., 2020; Deng S et al., 2019).

In a previous work we demonstrated that the deficiency of autophagy exacervates $T$. cruzi infection in mice and correlated with a higher parasite load in macrophages obtained from these autophagy-deficient mice or in macrophages obtained from wild-type animals that were then treated with autophagy inhibitors (Casassa AF et al., 2019). Given that autophagy plays a protective role on T. cruzi infection in vivo and having into account that UA reduces the parasitemia levels in infected mice, we hypothetized that the anti-T. cruzi effects of UA could be related to an action on autophagy. The main objective of this work was to study the effect of ursolic acid in the intracellular cycle of $T$. cruzi in both macrophages and cardiac cells and to describe its possible mechanism of action.

\section{Materials and Methods}

\subsection{Reagents}

Dulbecco's Modified Eagle Medium (D-MEM), penicillin and streptomycin were obtained from Gibco BRL/Life technologies. The polyclonal rabbit anti-LC3 antibody, the non-polar fluorescent probe 5-chloromethyl-2 ', 7'-dichlorodihydrofluorescein diacetate (H2DCFDA) acetyl ester and Griess reagent and Ursolic acid were purchased from Sigma-Aldrich. The polyclonal mouse anti- $\beta$ TUBULIN was obtained from Developmental Studies Hybridoma Bank. A polyclonal mouse antibody against T. cruzi was obtained from infected C57 mouse and polyclonal rabbit antibody against T. cruzi was kindly provided by Dr. David Engman (Northwestern University, Chicago, IL). The secondary antibody Cy3-conjugated anti-Goat IgG was purchased from Jackson ImmunoResearch Laboratories. The secundary antibody Alexa 488 was obteined from ThermoFisher. The DNA marker Hoechst 33342 and Lysotraker red were purchased from Life Technologies. The fetal bovine serum (FBS) was purchased from Natocor. Red DQ-BSA was obteined from Invitrogen. In Situ Cell Death Detection Kit, Fluorescein was obtained from Roche. The Nitrocellulosa from Amersham, Pittsburgh, PA.

\subsection{Amount of Amastigotes}

Raw macrophages, Bone marrow macrophages from C57 Wt and KD mice (Beclin -/+, deficient in the autophagic pathway) and $\mathrm{H} 9 \mathrm{C} 2$ cells were infected with trypomastigotes of strain $\mathrm{Y}$ (IFI) or YGFP (western blot and flow cytometry) with 10 parasites per cell (MOI 10), for 24 hours, then it was washed to eliminate the parasites that did not infect and fresh medium was added without or with 5 or $10 \mathrm{uM}$ of ursolic acid for 24,48 or 72 additional hours. Then the amastigotes were identified by immunofluorescence, western blot or flow cytometry.

For detection by immunopluorescence, the Cells were fixed with $4 \%$ paraformaldehyde in PBS for $15 \mathrm{~min}$ at room temperature, washed with PBS, and blocked with $50 \mathrm{mM} \mathrm{NH} 4 \mathrm{Cl}$. Subsequently, cells were permeabilized with $1 \%$ saponin in PBS containing $1 \%$ bovine serum albumin (BSA), and then were subjected to indirect immunofluorescence to detect amastigote of T. cruzi using a specific antibody and then were mounted with Mowiol 4-88 Reagent more Hoechst and examined by confocal 
microscopy, using an Olympus Confocal FV1000 microscope (Japan) and processed with the program FV10-ASW 1.7.

For analysis by Wester blot the cells were infected with tripomastigotes of Y-GFP strain, them the cell were lysed with sample buffer and protein samples were run on a $10 \%$ polyacrylamide gel and transferred to nitrocellulose membranes. The membranes were blocked $\mathrm{ON}$ in Blotto at $4^{\circ} \mathrm{C}$ (\% non-fat milk, 0.1\% Tween 20, and PBS), washed twice with PBS and incubated with a primary antibody T. cruzi (1:800 dilution) followed by a peroxidase-conjugated secondary antibody (1:10,000 dilution). Anti-TUBULIN (1:300 dilution) was used to detect TUBULIN as a loading control. The corresponding bands were detected using an enhanced chemiluminescence detection kit (Amersham, RPN2109) and the band was detected using Fujifilm LAS-4000.

For detection by flow cytometry the cells were infected with tripomastigotes of Y-GFP strain, them the cell were collected and their GFP fluorescence level was tested using BD Accuri C6 Pluss Flow Cytometer.

\subsection{Obtaining Macrophages Derived from Bone Marrow}

The bone marrow was obtained from the femur bones of C57BL / $6 \mathrm{Wt}$ and KD (Beclin +/-) mice, resuspended in cold D-Men, containing $40 \mu \mathrm{g} / \mathrm{ml}$ of gentamicin, following standard procedures. These bone marrow progenitor cells were recovered in $100 \mathrm{~mm}$ plates, containing 10\% FBS, $40 \mu \mathrm{g} /$ ml gentamicin, $2 \mathrm{mM}$ L-glutamine, and conditioned medium derived from 30\% L929 cell culture, for 4 days. Then, they were washed and the same medium was added for an additional 6 days. Finally the cells were typed using the markers F480 and CD11b.

\subsection{LC3 Detection}

Autophagy was induced by amino acid starvation. Raw macrophages and $\mathrm{H} 9 \mathrm{C} 2$ cells grown in 6 or 24-well plates were washed three times with PBS and incubated with control medium or with $0.5 \mathrm{ml}$ of Earle's balanced salt solution (starvation medium) at $37^{\circ} \mathrm{C}$ for $2 \mathrm{~h}$ in the presence or absence of drugs (wortmannin $100 \mathrm{nM}$ or UA $10 \mathrm{uM}$ ).

For detection by immunopluorescence, the Cells were fixed with $3 \%$ paraformaldehyde in PBS for $15 \mathrm{~min}$ at room temperature, washed with PBS, and blocked with $50 \mathrm{mM} \mathrm{NH} 4 \mathrm{Cl}$. Subsequently, cells were permeabilized with $1 \%$ saponin in PBS containing $1 \%$ bovine serum albumin (BSA), and then were subjected to indirect immunofluorescence to detect endogenous LC3 using a specific antibody and then were mounted with Mowiol 4-88 Reagent and examined by confocal microscopy, using an Olympus Confocal FV1000 microscope (Japan) and processed with the program FV10-ASW 1.7. The percentage of cells with more than 5 or $10 \mathrm{LC} 3$ dots/cell was determined. Confocal images of 10 random fields were quantified, representing around 100 cells per experiment. Data are presented as mean values and error bars indicate the SEM from at least three independent experiments. Statistical calculations (Student and Tukey's tests, significant differences were ${ }^{*} p<0.05 ;{ }^{* *} p<0.01$ ) were made using Kyplot statistica software and graphs were plotted with Microsoft Power Point.

For analysis by Wester blot the cells were lysed with sample buffer and protein samples were run on a $12.5 \%$ polyacrylamide gel and transferred to nitrocellulose membranes. The membranes were blocked ON in Blotto at $4^{\circ} \mathrm{C}$ (\% non-fat milk, $0.1 \%$ Tween 20, and PBS), washed twice with PBS and incubated with a primary antibody anti- LC3 (1:800 dilution) followed by a peroxidaseconjugated secondary antibody (1:10,000 dilution). Anti-TUBULIN (1:300 dilution) was used to detect TUBULIN as a loading control. The corresponding bands were detected using an enhanced chemiluminescence detection kit (Amersham, RPN2109) and the band was detected using Fujifilm LAS-4000.

\subsection{DQ-BSA Labeling}

Raw macrophages that overexpress GFP-LC3 grown in 24-well plates were washed three times with PBS and incubated with control medium at $37^{\circ} \mathrm{C}$ for $2 \mathrm{~h}$ in the presence or absence of UA $10 \mathrm{uM}$. Thirty minutes before the end of the reaction, $10 \mu \mathrm{g} / \mathrm{ml}$ of dequenched BSA was added. This 
compound emitted red fluorescence after BSA hydrolysis into small peptides in lysosomes, thus identifying degradative compartments. Them the cells were fixed with $3 \%$ paraformaldehyde in PBS for $15 \mathrm{~min}$ at room temperature, washed with PBS, and blocked with $50 \mathrm{mM} \mathrm{NH} 4 \mathrm{Cl}$ in PBS, and then were mounted with Mowiol 4-88 Reagent more hoechst and examined by confocal microscopy, using an Olympus Confocal FV1000 microscope (Japan) and processed with the program FV10-ASW 1.7.

\subsection{Lysotracker Labeling}

Raw macrophages that overexpress GFP-LC3 grown in 24-well plates were washed three times with PBS and incubated with control medium at $37^{\circ} \mathrm{C}$ for $2 \mathrm{~h}$ in the presence of lysotracker- red and the presence or absence of UA $10 \mathrm{uM}$. Them the cells were fixed with $3 \%$ paraformaldehyde in PBS for $15 \mathrm{~min}$ at room temperature, washed with PBS, and blocked with $50 \mathrm{mM} \mathrm{NH} 4 \mathrm{Cl}$ in PBS, and then were mounted with Mowiol 4-88 Reagent more hoechst and examined by confocal microscopy, using an Olympus Confocal FV1000 microscope (Japan) and processed with the program FV10-ASW 1.7.

On the other hand, Raw macrophages were infected with trypomastigotes of strain $Y$ with 10 parasites per cell (MOI 10), for 24 hours, then it was washed to eliminate the parasites that did not infect and fresh medium was added without or with $10 \mathrm{uM}$ of ursolic acid for 24 additional hours. Two hours before the end of the reaction, lysotraker red was added. Them the cells were fixed with 3\% paraformaldehyde in PBS for 15 min at room temperature, washed with PBS, and blocked with $50 \mathrm{mM} \mathrm{NH4Cl}$ in PBS, and then were mounted with Mowiol 4-88 Reagent more hoechst and examined by confocal microscopy, using an Olympus Confocal FV1000 microscope (Japan) and processed with the program FV10-ASW 1.7.

\subsection{Transmission Electron Microscopy}

Raw macrophages were infected with trypomastigotes of strain $Y$ with 10 parasites per cell (MOI 10), for 24 hours, then it was washed to eliminate the parasites that did not infect and fresh medium was added without or with $10 \mathrm{uM}$ of ursolic acid for 24 additional hours.

Briefly, cello or parasites were fixed with $2 \%$ glutaraldehyde (Ted Pella) in PBS for $2 \mathrm{~h}$ at $4^{\circ} \mathrm{C}$, washed three times with PBS pH 7.2 and subsequently treated with $1 \%$ osmium tetroxide (Ted Pella) for $2 \mathrm{~h}$ at $4 \hat{E} C$. In a next step, samples were washed again with PBS and sequentially dehydrated in solutions with increasing concentrations of acetone. Finally, samples were included in the epoxy resin (Spurr) and ultrathin sections in an ultramicrotome Leica Ultracut $\mathrm{R}$ were performed. Sections were contrasted with uranyl acetate / acetone for $3 \mathrm{~min}$, washed with distilled water and colored with lead citrate for 2 min before observation with the Zeiss 900 electron microscope.

\subsection{ROS Measurement}

Raw macrophages were infected with trypomastigotes of strain $Y$ with 10 parasites per cell (MOI 10), for 24 hours, then it was washed to eliminate the parasites that did not infect and fresh medium was added without or with $10 \mathrm{uM}$ of ursolic acid for 24 additional hours. We use the non-polar fluorescent probe acetyl ester of 5-chloromethyl-2', $7^{\prime}$-dichlorodihydrofluorescein diacetate (H2DCFDA), which crosses membranes freely, and after the action of cellular esterases it is trapped in the intracellular medium in the form of a derivative not fluorescent. Upon dissociation of acetate groups by intracellular esterases and oxidation, non-fluorescent H2DCFDA is converted to 2 ', 7'dichlorofluorescein (DCF), which is highly fluorescent, in the presence of an oxidizing environment. This fluorescence was detected by spectrofluorometry at $507 \mathrm{~nm}$ of excitation and $530 \mathrm{~nm}$ of emission.

\subsection{Griess Reaction}

Raw macrophages were infected with trypomastigotes of strain $\mathrm{Y}$ with 10 parasites per cell (MOI 10), for 24 hours, then it was washed to eliminate the parasites that did not infect and fresh medium was added without or with $10 \mathrm{uM}$ of ursolic acid for 72 additional hours. Subsequently, we follow the manufacturer's instructions and measure fluorescence by spectrophotometry. 


\subsection{Epimastigotes Viability}

Epimastigotes of the $\mathrm{Y}$ strain were grown in diamosn medium alone or with the addition of 5 , $10,12.5,25,50$ and $100 \mathrm{uM}$ of UA at $28^{\circ} \mathrm{C}$ for 24 hours. A small sample was extracted and live epimastigotes were counted in a Neubauer chamber. To calculate the inhibitory concentration 50 (IC50) and the graphs, the Microsoft Excel program was used.

\subsection{Tripomastigotes Viability}

Trypomastigotes (1,000,000 for each condition) of the $Y$ strain were incubated for $0.1 .5,3.5,6$ and 24 hours at $4^{\circ} \mathrm{C}$ in D-Men medium alone or more than 10 or 50 uM UA. At different times, a small sample was extracted and live epimastigotes were counted in a Neubauer chamber. To calculate the half maximal effective concentration (EC50) and the graphs, the Microsoft Excel program was used.

\section{Results and Discussion}

\subsection{Effect of Ursolic Acid on Amastigotes of T. cruzi}

To analyse the possible effect of ursolic acid on the intracellular infection of T. cruzi we infected macrophages derived from bone marrow (BMM), Raw macrophages and $\mathrm{H} 9 \mathrm{C} 2$ cells (rat cardioblasts) with trypomastigotes of the T. cruzi Y or Y-GFP strain for $24 \mathrm{~h}$, and treated with 5 or $10 \mu \mathrm{M}$ of UA for an additional time of 24,48 , or 72 hours. Subsequently, we evaluated the amount of amastigotes present in the treated cells by either confocal microscopy or western blot in comparison with infected cells maintained in control medium (Figure 1), A lower detection of T. cruzi amastigotes was observed by confocal microscopy in Raw cells as well as in BMM at $24 \mathrm{~h}$ of UA treatment (Figure 1A,C). Quantitative data showed that UA reduced the number of amastigotes/ cell in both types of macrophages being significant for the Y-GFP amastigotes observed in BMM (Figure 1B,D) On the other hand, we studied the amount of amastigotes in Raw macrophages at different times of treatment by western blot, by detection of the GFP protein present in the parasites with an anti-GFP specific antibody and observed a marked reduction of the infection mainly at the later 48 and $72 \mathrm{~h}$ of treatment (Figure 1E,G) We also detected amastigotes by flow cytometry (Figure 1I). 
A

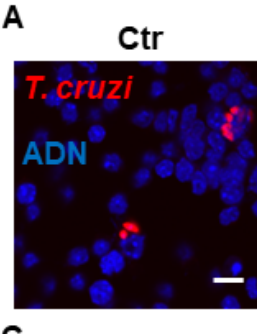

C

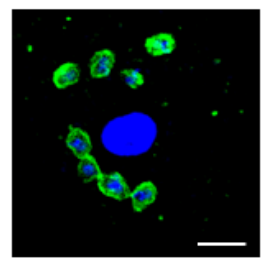

$\mathbf{E}$
UA
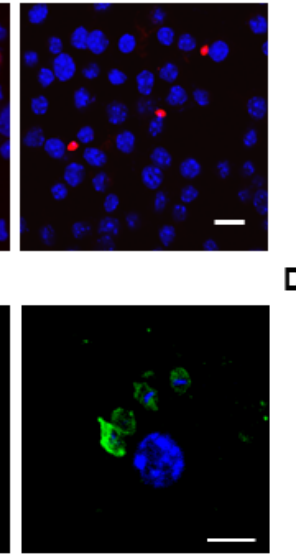

B

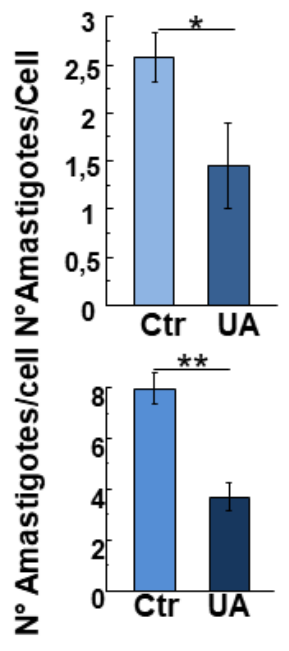

$\mathbf{F}$
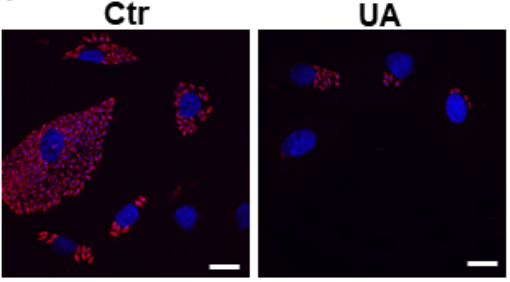

I
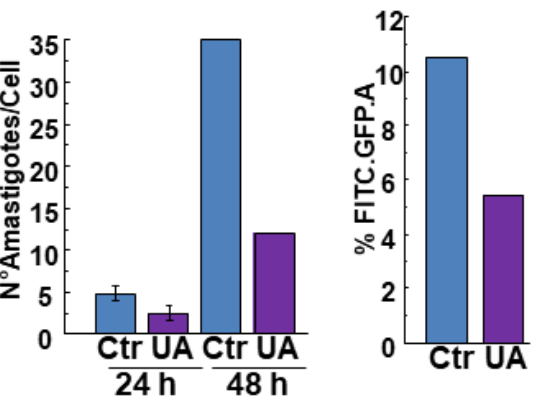

Figure 1. Effect of UA on the amount of intracellular amastigotes. Raw macrofagos, BMM or H9C2 cells infected with T. cruzi Y or Y-GFP strain for $24 \mathrm{~h}$ and then treated for an additional 24, 48 or $72 \mathrm{~h}$ under different conditions. (A) Confocal images showing amastigotes of T. cruzi (red) in Raw macrophages under the indicated conditions. Scale bars: $10 \mu \mathrm{m}$. (B) Quantification of the number of amastigotes per cell. Data represent the mean \pm SEM of at least three independent experiments (number of counted cells $\approx 100$ ). ${ }^{*}<0.05$, Tukey's test. (C) Confocal images showing amastigotes of $T$. cruzi (Green) in BMM under the indicated conditions. Scale bars: $10 \mu \mathrm{m}$. (D) Quantification of the number of amastigotes per cell. Data represent the mean \pm SEM of at least three independent experiments (number of counted cells $\approx 100$ ). ${ }^{* *}<0.01$, Tukey's test. (E) Representative immunoblots are depicted.(F) Densitometry was performed using NIH ImageJ. The GFP /TUBULIN were calculated. Data represent the mean \pm SEM of four independent experiments. (G) Confocal images showing amastigotes of T. cruzi (red) H9C2 under the indicated conditions. Scale bars: $10 \mu \mathrm{m}$. (H) Quantification of the number of amastigotes per cell. Data represent the mean \pm SEM of at least three independent experiments (number of counted cells $\approx 100$ ). (I) Quantification of the the FITC.GFP.A fluorescence intensity of $\mathrm{H} 9 \mathrm{C} 2$ cells infected with trypomastigotes of the Y-GFP strain for $24 \mathrm{~h}$ and then treated for an additional $24 \mathrm{~h}$ under the different conditions.

Taken together, these data demonstrated that UA impairs the intracellular cycle of T. cruzi resulting in a reduction in the amount of amastigotes present in the host cell cytosplasm at later times of infection. The reduction of parasitemia peak in mice infected with T. cruzi by UA previously observed by other authors (Daniele da Silva Ferreira, et al. 2013) could be a result of the action of UA 
to clear the amastigotes on infected cells. However, we did not discard other possible mechaisms as a direct toxic effect of UA on T. cruzi.

\subsection{Ursolic Acid Induces Autophagy and Xenophagy of T. cruzi Amastigotes}

In a previous work from our laboratory we demonstrated that autophagy plays a protective effect in a mice model of $T$. cruzi infection. We observed that mice deficient in autophagy developed a more aggressive infection and an earlier death of individuals compared with autophagy-competent mice. This study also showed that macrophages from deficient mice or control macrophages treated with autophagy inhibitors displayed less capacity to clear the amastigotes by the process of xenophagy (Casassa AF, et al. 2019). Xenophagy, the process of capture of pathogens in the cytoplasm of host cells, is a class of specific autophagy that belong to the repertorie of the innate immune responses activated in phagocytic cells against intracellular microorganisms (Sharma V et al.,2018). Due to there is evidence that ursolic acid is an inducer of the autophagy (Leng S, et al. 2016), we next asked if the action of this compound in the clearance of amastigotes was produced by xenophagy.

To test this, we first studied the possible effect of UA on the autophagy response of Raw and $\mathrm{H} 9 \mathrm{C} 2$ cells. Cells were treated with UA for $2 \mathrm{~h}$ and the presence of autophagosomes was analyzed by detection of endogenous LC3 protein by IIF followed by confocal microscopy (see details in materials and methods) in comparison to cells subjected to different conditions of induction (Stv) or inhibition (Stv+Wort) of autophagy. As shown in the images of Figure 2A, there is a different number of autophasomes formed in response to each conditon and according to the class of cell assayed. The percentage of cells with more than 5 (for Raw cells) or 10 (for H9C2 cells) autophagosomes, indicative of an active autophagic response, significantly increased in cells under starvation (Stv) when compared with control cells. Presence of wortmannin impairs this response displaying a reduction in the values. Interestingly, similar to starvation, the treatment with UA increased the number of autophagosomes which also diminished in the presence of wortmannin. To confirm these data, we next detected the endogenous LC3 by western blot and observed an increment in the level of LC3-II in the presence of UA (Figure 2B). Together, these data showed that UA treatment produce a significant increment in the number of autophagosomes in both Raw macrophages as well as $\mathrm{H} 9 \mathrm{C} 2$ cells. Because both induction or inhibition of autophagy degradation results in an increase in the number of autophagosomes, to confirm the action of UA on autophagy we next treated Raw cells that overexpress GFP-LC3 with UA and then incubated with Lysotracker or DQ-BSA, markers of acidic and hydrolytic compartments, to study the nature of autophagosomes. As shown in the Figure 2C, many autophagosomes formed under UA treatment were decorated with GFP-LC3 and also were stained with lysotracker or DQ-BSA indicating their autolysosomal nature and confirming that treatment with UA induced a functional autophagy response. 
A
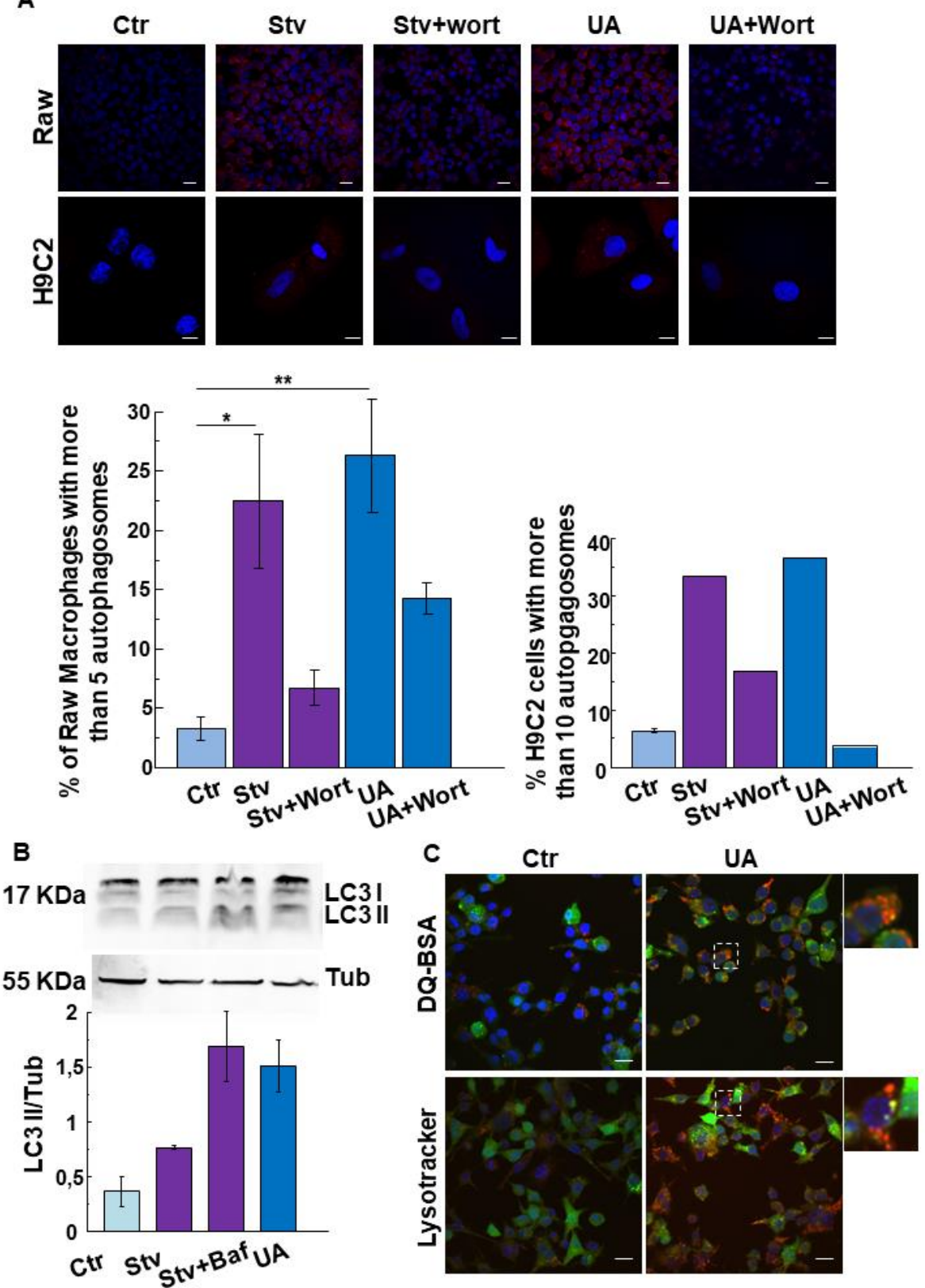

C Ctr

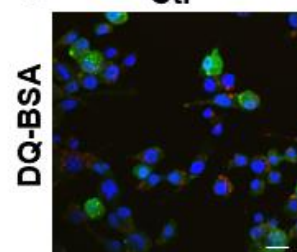

UA

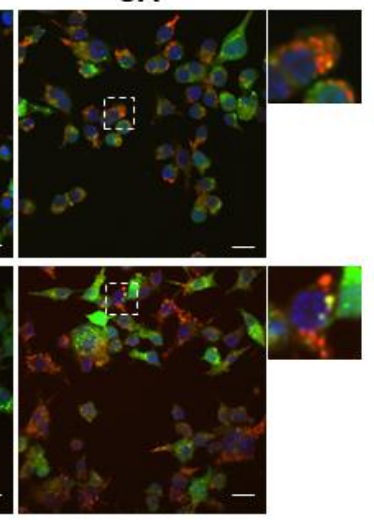

Figure 2. Ursolic acid stimulates autophagy in Raw macrophages and H9C2 Cells. Raw macrofages or H9C2 cells were incubated for $2 \mathrm{~h}$ in control or starvation medium (Stv) or in control medium supplemented with $10 \mu \mathrm{M}$ UA alone (UA) or in the presence of $100 \mathrm{nM}$ wortmannin (UA + Wort) or starvation medium (Stv) in the presence of $100 \mathrm{nM}$ wortmannin (Stv + Wort); $100 \mathrm{nM}$ bafilomycin A1 (Stv + Baf) as indicated under Materials and Methods. (A) Confocal images depict LC3 (red) distribution under the indicated conditions. Scale bars: $10 \mu \mathrm{m}$. Graph shows the percentage of cells with more than 5 puncta (Raw macrofages) or 10 puncta per cell (H9C2 cells) in each condition. Data represent the mean \pm SEM of at least three independent experiments (number of counted cells $\approx 100$ ). $*<0.05,{ }^{*}<0.01$. Tukey's test. (B) Upper panel: Representative immunoblot of three experiments corresponding to LC3 detection is depicted. Lower panel: quantification of the LC3II/Tub ratio. Data are representative of three independent experiments. (C) Raw macrofages overexpressing GFP-LC3 were grown in control medium in the presence or absence of $10 \mu \mathrm{M} U \mathrm{UA}$ (UA) for $2 \mathrm{~h}$. Confocal images depict GFP-LC3 and DQ-BSA or Lysotracker distribution under the indicated conditions. Scale bars: $10 \mu \mathrm{m}$.

Next we studied if UA induced autophagy was responsible for the elimination of amastigotes in the host cell cytoplasm. Raw macrophages or H9C2 cells were infected with T. cruzi for $24 \mathrm{~h}$ and 
treated with $10 \mu \mathrm{M}$ UA for $24 \mathrm{~h}$ more. After fixation cells were subjected to double IIF to detect the distribution of endogenous LC3 and T. cruzi amastigotes. Confocal images showed the presence of LC3 sourronding parasites in the UA condition in comparation with control non-treated cells for both cell types (Figure 3A). Furthermore, compared to control, UA increased the recruitment of lysotracker positive compartments in the vicinity of amastigotes in macrophagues (Figure 3B). On the other hand, we studied the ultra-structure of infected macrophagues by transmission electron microscopy and observed the presence of membrane structures surrounding the parasites in both control as well as under UA treatment (Figure 3C). These data confirm that UA induced autophagy and promotes the clearece of amastigotes in the cytoplasm of host cells.

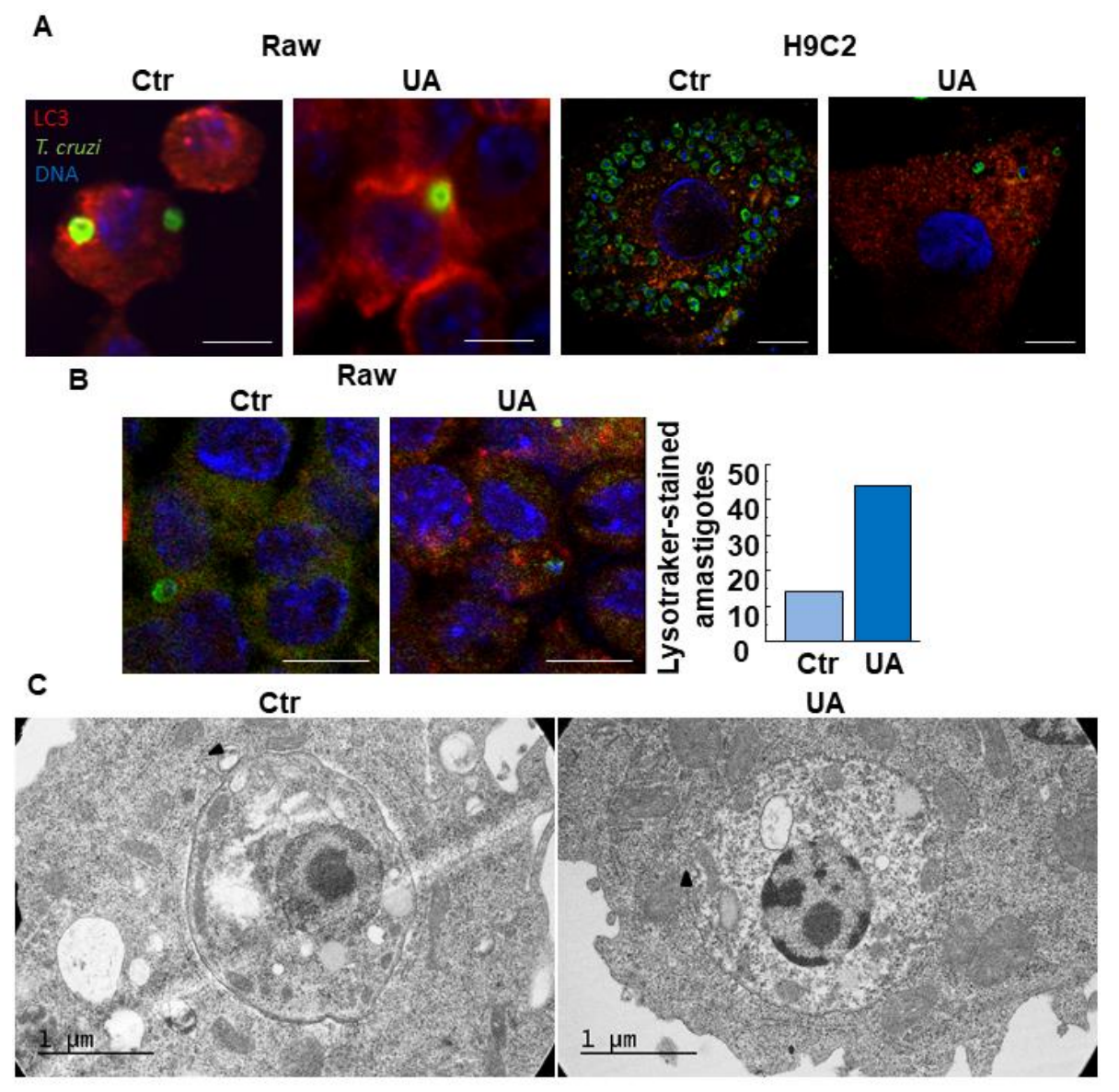

Figure 3. Evaluation of xenophagy. Raw macrofagos or H9C2 cells infected with T. cruzi Y strain for $24 \mathrm{~h}$ and then treated for an additional $24 \mathrm{~h}$ under different conditions. (A) Confocal images depicting LC3 (red) localization around T. cruzi amastigotes under the indicated conditions. Scale bars: $10 \mu \mathrm{m}$. (B) Images depict Lysotracker labeling around T. cruzi amastigotes under the indicated conditions. Scale bars: $10 \mu \mathrm{m}$. The graph shows the quantification of the percentage of amastigotes surrounded by lysotracker (C) Images show intracellular amastigotes by TEM.

\subsection{Exploring Other Mechanisms of Action of Ursolic Acid Against T. cruzi}

In other set of experiments, we performed primary cultures of BMM obtained from Beclin-1 heterocygous knock out mice (KD) and studied the level of infection in the absence or presence of UA in comparison with cells obtained from wild type animals. Interestingly, the number of amastigotes in the cells was also reduced in the autophagy-deficient macrophages (Figure 4A), indicating that different mechanisms other than autophagy have been activated by UA. In this way, we decided to explore the action of UA in the production of ROS using the non-polar fluorescent probe H2DCFDA, and NO by the Griess reaction (see details in materials and methods). The generations of these compounds are one of the main mechanisms of the innate immune response of macrophages against T. cruzi (Dos-Santos AL et al., 2016). Our results showed that UA have a 
tendency to increase the ROS generation on infected cells whereas NO not displayed differences to controls (Figure 4B,C). Unexpectedly, the UA-induced increase in ROS level only occurred in infected cells while the controls remain similar. These data suggested in some way the participation of inflamasomes in this process, in agreement with the evidence that the integration of inflammasome activation, autophagy and cell death is fundamental for the elimination of pathogens. (Krakauer T, 2019). We also analyzed a possible direct cytotoxic effect of UA on T. cruzi by studying the cell viability of epimastigotes and trypomastigotes treated with increased concentrations of UA for $24 \mathrm{~h}$. As shown in the Figure 4D and E while the IC50 for epimastigotes was 101.02+/-9.91 $\mu \mathrm{M}$, the EC50 for trypomastigotes was $5.73+/-0.02$. These data indicate that UA displayed high toxicity to this parasitic form of T. cruzi. More experiments are needed to evaluate the toxicity of UA in amastigotes.
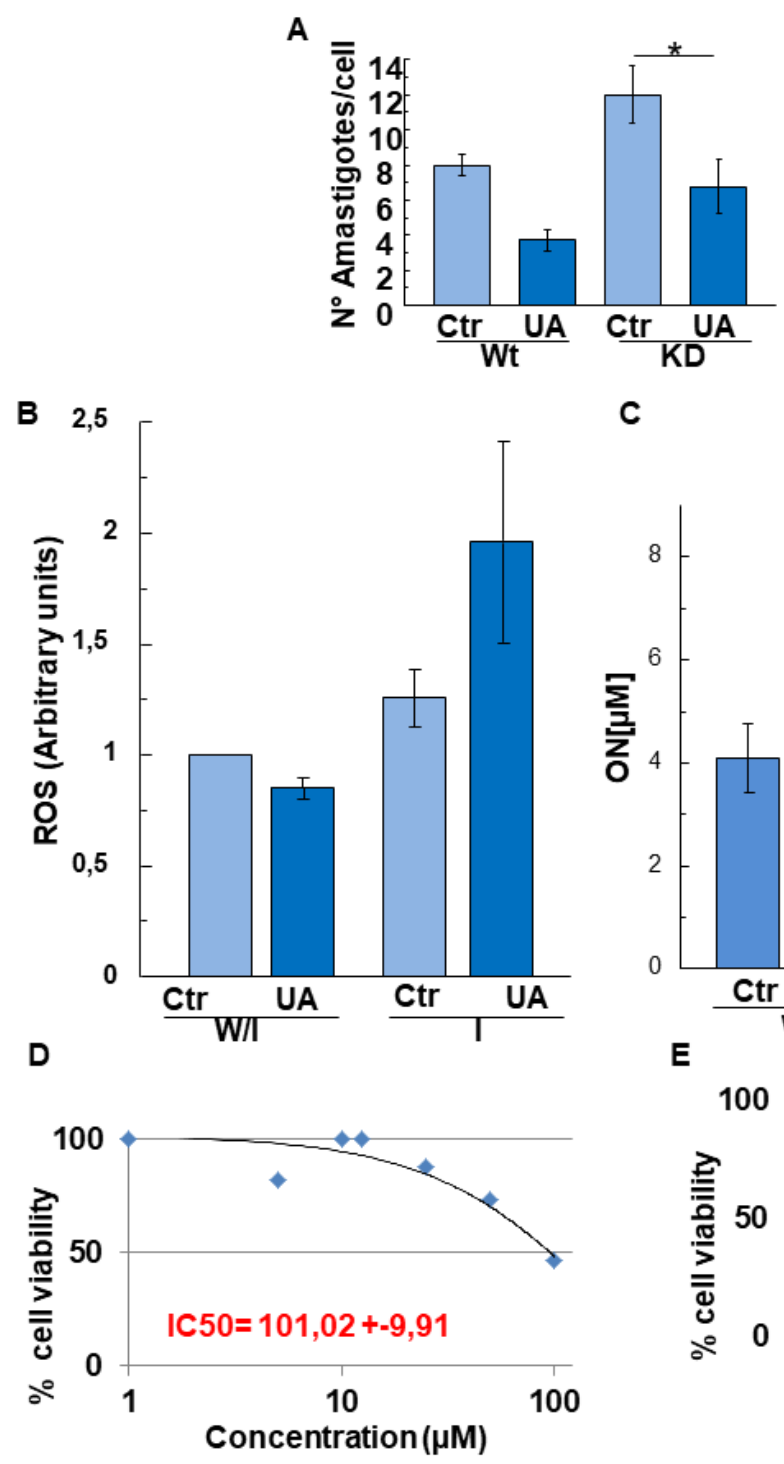

C

D

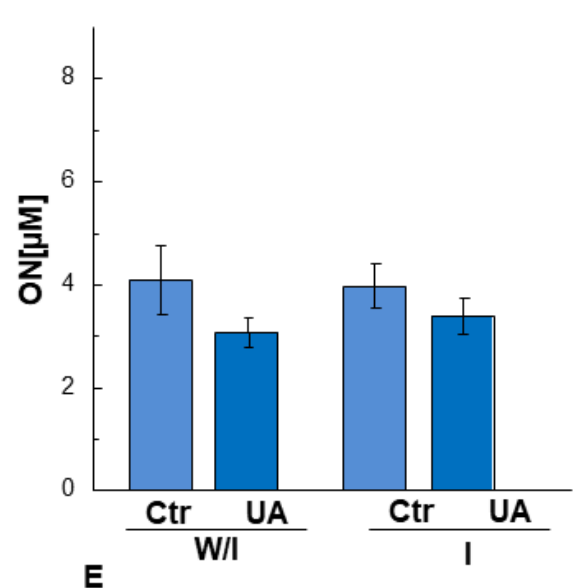

E

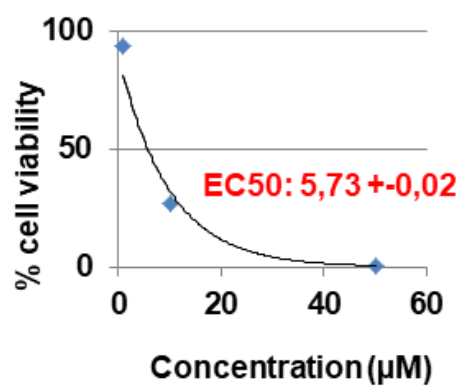

Figure 4. Raw macrophage oxidative response and direct action of UA in the different stages of $T$. cruzi (A) BMM wt r Beclin +/- (KD) infected with T. cruzi Y strain for $24 \mathrm{~h}$ and then treated for an additional $24 \mathrm{~h}$ under different conditions. The graph shows the quantification of the number of amastigotes per cell. Data represent the mean \pm SE- M of at least three independent experiments (number of counted cells $\approx 100$ ). ${ }^{*}<0.05$, Tukey's test. (B) Raw macrofages infected with T. cruzi $Y$ strain for $24 \mathrm{~h}$ and then treated for an additional $24 \mathrm{~h}$ under different conditions. The graph shows the quantification of the ROS. Data the mean \pm the standard deviation of two independent experiments. (C) Raw macrofages infected with T. cruzi Y strain for $24 \mathrm{~h}$ and then treated for an additional $72 \mathrm{~h}$ under different conditions. The graph shows the quantification of the NO, in the cell supernatant. The graph shows the data the mean, \pm the standard deviation of three independent experiments. (D) Epimastigotes were grown in different concentrations of UA for 24 hours at $28^{\circ} \mathrm{C}$. 
The graph shows the quantification of IC50. (E) The trypomastigotes were treated at different concentrations of UA for 24 hours at $4^{\circ} \mathrm{C}$. The graph shows the quantification of the EC50.

\section{Conclusions}

We conclude that UA has an anti-T. cruzi activity which is executed by different mechanisms that involved autophagy induction, xenophagy and oxidative responses. We also found that UA has a direct tripanocidal action on T. cruzi trypomastigotes.

\section{References}

1. Chagas disease: an overview of clinical and epidemiological aspects. Maria Carmo Pereira Nunes, Wistremundo Dones, Carlos A Morillo, Juan Justiniano Encina, Antônio Luiz Ribeiro, Council on Chagas Disease of the Interamerican Society of Cardiology. J Am Coll Cardiol. 2013 Aug 27; 62(9):767-76.

2. Chagas disease as example of a reemerging parasite. Jeannette Guarner. Semin Diagn Pathol. 2019 May; 36(3):164-169.

3. Facing Chagas disease. João Carlos Pinto Dias. Rev Soc Bras Med Trop. May-Jun 2017; 50(3):285-286.

4. L-arginine metabolism during interaction of Trypanosoma cruzi with host cells. Gonzalo Peluffo, Lucía Piacenza, Florencia Irigoín, María Noel Alvarez, Rafael Radi. Trends Parasitol. 2004 Aug; 20(8):363-9.4- In vivo activity of ursolic and oleanolic acids during the acute phase of Trypanosoma cruzi infection. Daniele da Silva Ferreira, Viviane Rodrigues Esperandim, Miriam Paula Alonso Toldo, Christian Collins Kuehn, José Clóvis do Prado Júnior, Wilson Roberto Cunha, Márcio Luís Andrade e Silva, Sérgio de Albuquerque. Exp Parasitol. 2013 Aug; 134(4):455-9.

5. Trypanosomatid Infections: How Do Parasites and Their Excreted-Secreted Factors Modulate the Inducible Metabolism of 1-Arginine in Macrophages? Philippe Holzmuller, Anne Geiger, Romaric Nzoumbou-Boko, Joana Pissarra, Sarra Hamrouni, Valérie Rodrigues, Frédéric-Antoine Dauchy, Jean-Loup Lemesre, Philippe Vincendeau, Rachel Bras-Gonçalves. Front Immuno. 2018 Apr 20; 9:778.

6. Autophagy plays a protective role against Trypanosoma cruzi infection in mice. Ana Florencia Casassa, María Cristina Vanrell, María Isabel Colombo, Roberta A Gottlieb, Patricia Silvia Romano. Virulence. 2019 Dec; 10(1):151-165.

7. Ursolic Acid-Based Derivatives as Potential Anti-Cancer Agents: An Update. Vuyolwethu Khwaza, Opeoluwa O Oyedeji, Blessing A Aderibigbe. Int J Mol Sci. 2020 Aug 18; 21(16):5920.

8. Ursolic Acid Protects Against Proliferation and Inflammatory Response in LPS-Treated Gastric Tumour Model and Cells by Inhibiting NLRP3 Inflammasome Activation. Zixi Chen, Qiaoli Liu, Zhaowei Zhu, Fenfen Xiang, Mengzhe Zhang, Rong Wu, Xiangdong Kang. Cancer Manag Res. 2020 Sep 11; 12: 8413-8424.

9. Ursolic acid: A novel antiviral compound inhibiting rotavirus infection in vitro. M J Tohmé, M C Giménez, A Peralta, M I Colombo, L R Delgui. Int J Antimicrob Agents. 2019 Nov; 54(5):601-609.

10. Evaluating the Potential of Ursolic Acid as Bioproduct for Cutaneous and Visceral Leishmaniasis. Pablo Bilbao-Ramos, Dolores R Serrano, Helga Karina Ruiz Saldaña, Juan J Torrado, Francisco Bolás-Fernández 1, María Auxiliadora Dea-Ayuela. Molecules. 2020 Mar 19; 25(6):1394.

11. Therapeutic Potential of Ursonic Acid: Comparison with Ursolic Acid. Juhyeon Son, Sang Yeol Lee. Biomolecules. 2020 Nov 2; 10(11):E1505.

12. Ursolic acid promotes apoptosis, autophagy, and chemosensitivity in gemcitabine-resistant human pancreatic cancer cells. Ji-Hua Lin, Sheng-Yi Chen, Chi-Cheng Lu, Jer-An Lin, Gow-Chin Yen. Phytother Res. 2020 Aug; 34(8):2053-2066.

13. Targeting autophagy using natural compounds for cancer prevention and therapy. Shuo Deng, Muthu K Shanmugam, Alan Prem Kumar, Celestial T Yap, Gautam Sethi, Anupam Bishayee. Cancer. 2019 Apr 15; 125(8):1228-1246.

14. The autophagic pathway is a key component in the lysosomal dependent entry of Trypanosoma cruzi into the host cell. Patricia S Romano ${ }^{1}$, María A Arboit, Cristina L Vázquez, María I Colombo. Autophagy. 2009 Jan; 5(1):6-18.

15. Polyamine depletion inhibits the autophagic response modulating Trypanosoma cruzi infectivity. María C Vanrell, Juan A Cueto, Jeremías J Barclay, Carolina Carrillo, María I Colombo, Roberta A Gottlieb, Patricia S Romano. Autophagy. 2013 Jul; 9(7):1080-93. 
Publisher's Note: MDPI stays neutral with regard to jurisdictional claims in published maps and institutional affiliations.

(C) 2020 by the authors. Licensee MDPI, Basel, Switzerland. This article is an open access article distributed under the terms and conditions of the Creative Commons Attribution (CC BY) license (http://creativecommons.org/licenses/by/4.0/). 